\title{
(6) OPEN ACCESS \\ Rheumatic heart disease among adults in a mining community of Papua, Indonesia: findings from an occupational cohort
}

\author{
Rodrigo Rodriguez-Fernandez, ${ }^{1,2}$ Rachel Amiya, ${ }^{2,3}$ Rosemary Wyber, ${ }^{4}$ \\ Wishnu Widdodow, ${ }^{5}$ Johnathan Carapetis ${ }^{4}$
}

${ }^{1}$ Freeport Public Health and Malaria Control, International SOS, Kuala Kencana, Papua, Indonesia

${ }^{2}$ NCD Asia Pacific Alliance, Tokyo, Japan

${ }^{3}$ Department of Family Nursing, University of Tokyo, Graduate School of Medicine, Tokyo, Japan

${ }^{4}$ University of Western

Australia, Telethon Kids Institute, Subiaco, Western

Australia, Australia

${ }^{5}$ Department of Cardiology and

Vascular Medicine, University

of Indonesia, Jakarta,

Indonesia

Correspondence to

Dr R Rodriguez-Fernandez, International SOS Freeport Industrial Public Health and Malaria Control, Jalan

Kertajasa 1, Kuala Kencana, Mimika, Papua 99920 .

Indonesia;

rod.rodriguez@ncdapa.org

Received 21 May 2015

Revised 1 July 2015

Accepted 7 July 2015

\section{CrossMark}

To cite: RodriguezFernandez R, Amiya R, Wyber $\mathrm{R}$, et al. Heart Asia 2015;7:1-5. doi:10.1136/ heartasia-2015-010641

\section{ABSTRACT}

Background Rheumatic heart disease (RHD) remains a significant cause of cardiovascular morbidity and mortality in developing countries such as Indonesia. Yet, despite being one of the most readily preventable chronic diseases, RHD has received scant research or policy attention, particularly in South-East Asia.

Aim To describe the pattern of RHD occurrence in a sample of presenting cases from an occupational cohort in Papua Province, Indonesia.

Methods Clinical records of 15608 mining workers ( $96.4 \%$ men, mean age $36.3 \pm 7.4$ years) were reviewed retrospectively to identify and extract data on all rheumatic fever (RF) and RHD cases admitted to two hospitals in Papua during 2008-2013. Collected data included basic demographics, employment history and echocardiographic findings.

Results 83 RHD cases (95.6\% men, mean age 39.6 \pm 12.5 years) and 3 RF cases were identified between 2008 and 2013. Increased RHD risk was observed among those aged 35-44 ( $\mathrm{HR}=3.60)$ and $45-68$ $(H R=4.46)$ years relative to the youngest age group $(p<0.01)$. RHD incidence density was 6.84 per 10000 person years of follow-up. Among cases, mitral stenosis was the most common valvular lesion at initial presentation (41.0\%), and $6.0 \%$ were multivalvular. Conclusions The prevalence of RHD in Papuan mining workers correlates with adult prevalence data in other populations with a high RHD burden, highlighting RHD as a significant health issue into adulthood. The late stage at which most patients presented points to a strong need for earlier intervention. Both primary and secondary preventive measures must be considered critical tools to prevent and reduce RHD burden, particularly among older age groups.

\section{INTRODUCTION}

Rheumatic fever (RF), and its most serious complication, rheumatic heart disease (RHD), continue to be major global health problems. In individuals with a presumed inherited susceptibility, infection with Streptococcus pyogenes (group A streptococcus) can precipitate RF-the precursor to RHD-as an abnormal autoimmune reaction. Damage to heart valves caused by RF is termed RHD. Subsequent group A streptococcus infections and recurrence of RF accelerate valve damage. Without intervention, RHD progresses to heart failure and increases the risk of cerebrovascular accident, bacterial endocarditis and arrhythmias. ${ }^{1}$
RF/RHD occur predominately in developing countries, home to $80 \%$ of all people living with the disease. ${ }^{2}$ However, RF and RHD also persist in developed countries, particularly within socioeconomically deprived and indigenous populations. ${ }^{3}$ More than 15.6 million people globally suffer from RHD, and 233000 die prematurely of the disease every year, ${ }^{3}$ although the true global prevalence is likely to be closer to 34 million. ${ }^{4}$ According to the 2010 Global Burden of Disease Study, the number of disability adjusted life years lost to RHD was as high as 10.1 million per year worldwide. ${ }^{5}$ It is the most important cause of acquired cardiovascular disease in children and young adults, and the most common cause of multivalvular disease in low and middle income countries. $^{3} 6$ Moreover, RHD imposes a substantial economic burden, further straining already overstretched health systems. ${ }^{2}$

Overall, the incidence and prevalence of RF and RHD show very significant global variation. ${ }^{7}$ The disease has been almost eradicated in developed settings with improved living conditions, nutrition, access to medical care and prevention policiesincluding both primary (treatment of sore throats) and secondary (long term administration of antibiotics) approaches-brought by economic and social development. $^{3}$ RHD disproportionately affects individuals in low and middle income countries, including Asia. ${ }^{7}$ Demographic trends, including poor access to health services and rural exodus, will likely contribute to a substantial rise in RHD in the next 20 years. $^{8}$

Previous studies in Asia have estimated a current RHD burden of 10.8-15.9 million patients, ${ }^{9}$ accounting for 356 000-524000 deaths per year. ${ }^{10}$ However, epidemiological data remain sparse and inconsistent. In Indonesia, what limited data currently exist suggest that RHD continues to be relevant. In West Java, 108 patients aged $17-75$ years were identified with RHD, with complications suggesting an advanced burden of the disease. ${ }^{11}$

Recent directives from WHO and the World Heart Federation have pledged a $25 \%$ reduction in the number of deaths due to non-communicable diseases by $2025 .^{12}$ RHD is a disease where this may be achievable, given the availability of relatively inexpensive, proven and effective control strategies that can lead to reductions in deaths. Yet attention to the problem is lacking, and there are currently no coordinated national control 
programmes in place to address the RF/RHD burden in Indonesia. Community and hospital based surveys provide important information about disease burden in adults, in whom RHD prevalence is ultimately the highest. ${ }^{3}$ Although perceived as a disease of childhood, RF/RHD cases in children of 5-14 years are likely to represent only $15-20 \%$ of the total burden within all age groups of vulnerable populations. ${ }^{2}$

The present study was undertaken to describe the distribution of valvular involvement in newly diagnosed adult RHD patients attending two healthcare facilities within an occupational cohort in Papua Province, Indonesia, a region facing some of the least favourable socioeconomic indicators nationally. ${ }^{13}$ Given the marked paucity of epidemiological research on RHD in Indonesia, even basic descriptive data can provide important preliminary insights regarding the characteristics of the disease burden and the underlying factors defining it.

\section{METHODS}

\section{Study population and setting}

This research forms part of the ongoing Cardiovascular Outcomes in the Papuan Population and Estimation of Risk (COPPER) study. The present study population comprised workers aged 18-68 years who were employed by a multinational mining company, from January 2008 to December 2013. Participants were first recorded into the health information system (HIS) at routine medical screening during their first year of employment. Medical screening procedures have remained constant throughout the past 6 years and consist of medical history taking, physical examination, electrocardiography, chest X-ray, spirometry, audiometry and basic blood panel.

The COPPER study is set in the Mimika District, in the south of Papua Province, Indonesia's largest and easternmost province. Overall health status in Mimika remains the lowest in Indonesia, and the problems of access to services mean that a high proportion of the population remains under served. ${ }^{13}$ The mining company operates one of the largest gold and copper mines in the world, and is the main employer in the district. The company directly supports a primary and secondary hospital within the contract of work area, five community healthcare clinics and several mine site health posts. The health posts and hospitals are managed by a private healthcare provider.

\section{Data collection}

A retrospective chart review of medical records from two clinic sites was undertaken, covering the period from 1 July 2008 to 31 December 2013. One site is located adjacent to Timika and the second within the remote mountainous area of the Papuan Highlands. As part of routine care, RHD cases were first identified based on clinical suspicion (eg, finding on clinical cardiac auscultation), either during an early medical checkup or because of symptoms, and referred to an on-site cardiologist. Examination was then performed by the cardiologist and an opinion rendered as to the presence or absence of clinical evidence of RHD. An on-site cardiologist performed Doppler echocardiographic studies on suspected RHD patients using a General Electric Vivid-i machine. Echocardiograms included parasternal long and short axis and apical three and four chamber views. If a diagnosis of RHD was confirmed, it was documented under the appropriate ICD-10 category within the HIS.

RHD cases diagnosed in this manner, along with RF cases, were then retrospectively identified from the electronic HIS with the use of diagnostic ICD-10 codes. RHD included all cases with established heart disease confirmed on echocardiography as being of rheumatic origin. Extracted data for these codes included basic demographics, duration of employment and echocardiographic findings. If cases presented on more than one occasion, only the first RHD presentation since the cohort inception in 2008 was considered.

\section{Statistical analysis}

Demographic and occupational characteristics were described for all participants at baseline. Incidence rates were calculated as the number of incident RHD cases over the number of persons at risk at the beginning of the assessed time period. For incidence density calculation, follow-up person years were calculated for study participants until RHD diagnosis, until 31 December 2013 for those uncensored or until the censoring date for those censored for other reasons, such as death or termination of employment.

Univariate analyses were completed with Kaplan-Meier methods to describe the cumulative probability of RHD over time and to graphically confirm the assumption of proportional hazards. Multivariate analyses were carried out using Cox proportional hazards regression models to assess the risk of RHD across different age strata, adjusting for gender and duration of employment. All statistical analyses were completed using SPSS 22.0 for Windows (IBM SPSS Inc, Chicago, Illinois, USA). Statistical significance was defined as $\mathrm{p}<0.05$.

\section{RESULTS}

In total, data were available for 15608 workers, 560 (3.6\%) of whom were women (table 1). Mean age among employees at cohort enrolment in July 2008 was $36.3 \pm 7.4$ years, with employee attrition rates ranging from $0.7 \%$ to $6 \%$ from year to year to December 2013, with a net population increase of $3 \%$ via new hires over that same period. Mean time at risk contributed by cohort members over the assessed period was 4.3 \pm 0.8 years.

The total number of RHD patients identified from the medical records review and included in this study was 83 . Mean age of RHD cases was $39.6 \pm 12.5$ years, slightly higher than the overall population mean, and the majority $(n=79,79.6 \%)$ were men (95.2\%). Women were disproportionately represented among the RHD cases relative to their proportion of the

Table 1 Baseline demographic characteristics of participants and identified rheumatic heart disease cases

\begin{tabular}{|c|c|c|}
\hline Characteristic & $\begin{array}{l}\text { All surveyed } \\
\text { participants } \\
(n=15608)\end{array}$ & $\begin{array}{l}\text { Identified RHD } \\
\text { cases }(n=83)\end{array}$ \\
\hline \multicolumn{3}{|l|}{ Gender (\%) } \\
\hline Male & 15048 (96.4) & 79 (95.2) \\
\hline Female & $560(3.6)$ & $4(4.8)$ \\
\hline \multicolumn{3}{|l|}{ Age $(\%)^{*}$} \\
\hline $18-29$ years & $3450(22.2)$ & $9(10.8)$ \\
\hline $30-34$ years & $3410(21.9)$ & $9(10.8)$ \\
\hline $35-39$ years & 3814 (24.5) & $23(27.7)$ \\
\hline $40-44$ years & 2839 (18.3) & $15(18.1)$ \\
\hline $45-68$ years & 2040 (13.1) & 27 (32.5) \\
\hline Mean $\pm S D$ & $36.3 \pm 7.4$ & $41.3 \pm 8.6$ \\
\hline $\begin{array}{l}\text { Duration of employment at cohort } \\
\text { enrolment (years) (median (IQR)) } \dagger\end{array}$ & $9(4)$ & $10(11)$ \\
\hline
\end{tabular}




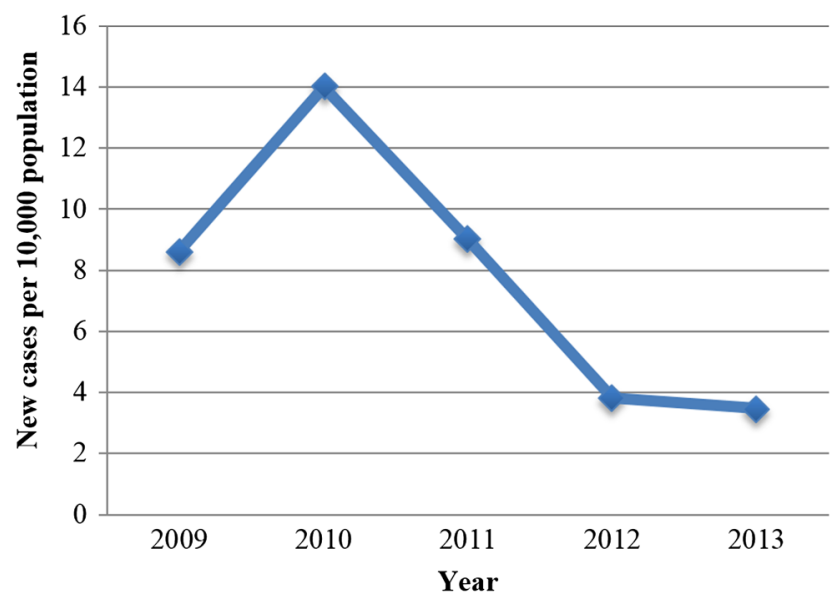

Figure 1 Cumulative incidence of rheumatic heart disease, 20092013. Points represent new cases per 10000 persons at risk per year.

population $(4.8 \%$ of RHD cases vs $3.6 \%$ of the total surveyed records) (table 1). Median number of years employed by the company prior to RHD diagnosis was 10 (IQR, 11) years. At the conclusion of the assessed period, $55.4 \%$ of those diagnosed with RHD remained in the mining company's workforce.

The overall prevalence of RHD recorded in 2008 was 2.52 cases per 1000 persons-2.48 and 3.70 per 1000 among men and women, respectively. The cumulative RHD incidence ranged from 3.83 in 2012 to 14.0 in 2009 . Although rates fluctuated somewhat over the years, particularly in 2010, the overall trend was decreasing (figure 1). The RHD incidence density rate was 6.84 per 10000 person years of follow-up.

Kaplan-Meier analyses revealed that the cumulative probability of RHD diagnosis increased at different rates across different age strata for all workers (figure 2). Results of log rank tests indicated that the probabilities of RHD diagnosis among those aged 35-44 years and among those aged 45-68 years were significantly greater than among those aged 18-34 years $(p<0.001)$. After adjustment for gender and employment

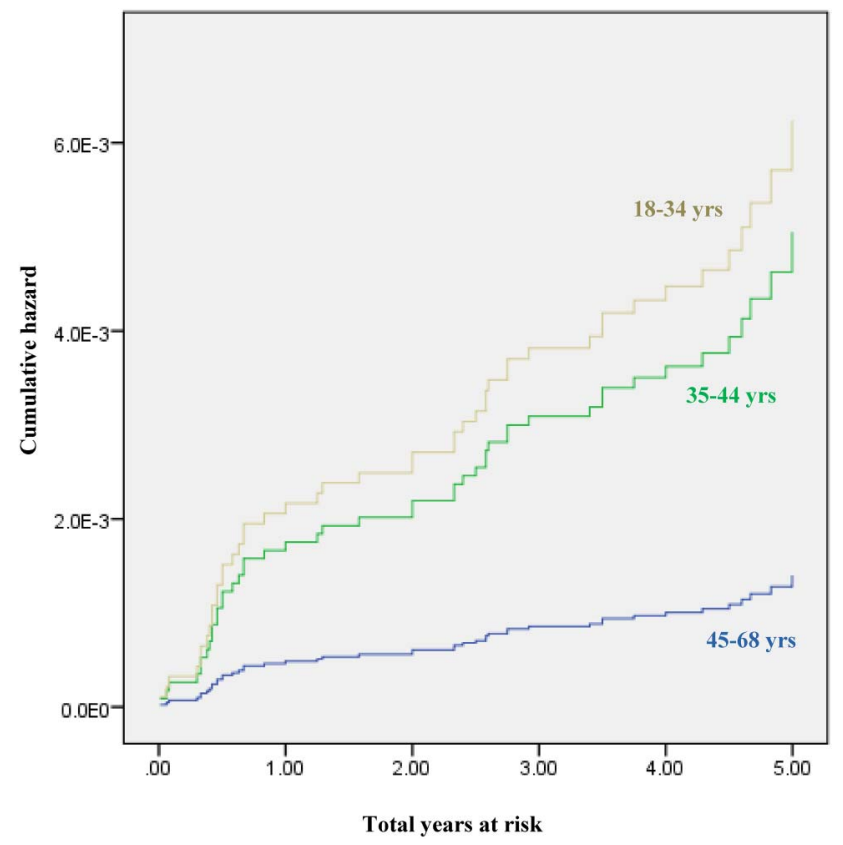

Figure 2 Kaplan-Meier hazard curve for cumulative probability of rheumatic heart disease diagnosis stratified by age category. duration, these trends remained significant; increased RHD risk was observed among those aged 35-44 years (HR 3.60, 95\% CI 1.54 to 8.45 ) and among those aged 45-68 years (HR 4.46, $95 \%$ CI 1.57 to 12.63 ) relative to the youngest age group.

Of the 83 cases with RHD, echocardiography findings were available for all cases. The most common valvular lesion was mitral stenosis, found in $41.0 \%(n=34)$ of cases, followed by mitral/aortic regurgitation, occurring in $20.5 \%(n=17)$ of cases each. Single valve involvement was more frequent than multiple valve involvement, with multivalvular disease identified in only $6.0 \%(n=5)$ of cases. The predominance of mitral stenosis was substantially more marked among those aged 18-34 years relative to the older age groups, while most instances of identified multivalvular involvement were in the older age groups (figure 3). The total number of RF patients identified from the medical records review and included in this study was three.

\section{DISCUSSION}

The prevalence of RHD in adult Papuan mine workers is consistent with adult prevalence data in other populations with a high RHD burden. ${ }^{3}$ Given the biases inherent in this study sample (ie, primarily male and employed), rates are likely to be even higher in the general population. The findings thus highlight that RF and RHD remain major health issues into adulthood. This points to a strong need for early public health intervention. Both primary and secondary preventive measures must be considered mainstay tools, particularly among older age groups.

Cumulative RHD risk was significantly higher among older age groups. Although the peak age of RF occurrence is typically during childhood, initial attacks of RF occurring in the fourth decade have been reported. ${ }^{14}$ Moreover, RHD risk in the present study may also be attributed to recurrent RF/RHD episodes, pre-2008 instances of which could not be ruled out. The results suggest that tailored interventions targeting older employees may be especially important in alleviating the RHD burden.

The most frequent valvular lesion among RHD cases was mitral stenosis, consistent with findings from previous studies carried out in Asia. ${ }^{15}{ }^{16}$ However, other studies have reported mitral regurgitation ${ }^{17} 18$ or combined mitral stenosis ${ }^{19} 20$ to be the predominant valvular presentation. The cause behind mitral valve tropism remains unclear. ${ }^{18}$ Mitral regurgitation was found in $20.5 \%$ of RHD cases in our population, while observed rates ranged between $18.4 \%$ and $42.9 \%$ in other studies. ${ }^{19} 20$ The $3.7 \%$ rate of tricuspid regurgitation found was slightly lower than findings from other Asian cohorts, with one study in India finding a $5.7 \%$ tricuspid regurgitation rate. ${ }^{20}$ Finally, aortic regurgitation was found in $20.5 \%$ of our RHD cases, compared with reported rates ranging between $3.1 \%$ and $47 \%$ in other studies. $^{15-17}$

Only three cases of RF were identified during this study, despite the fact that nearly $25 \%$ of the total workforce were aged 18-29 years (table 1). Given the increasing prevalence of RHD with age, it is likely that a number of subclinical or undiagnosed cases of RF occurred in childhood, early adolescent or young adulthood in this cohort. Diagnosing these cases of RF would potentiate early initiation of secondary prophylaxis and potentially avert development of severe RHD.

A decreased incidence of RHD has been observed with the advent of effective antistreptococcal therapy, particularly in developed countries. In our study population, a general decreasing trend was also observed in cumulative incidence over the past 5 years. Measured annually, the mean number of incident 


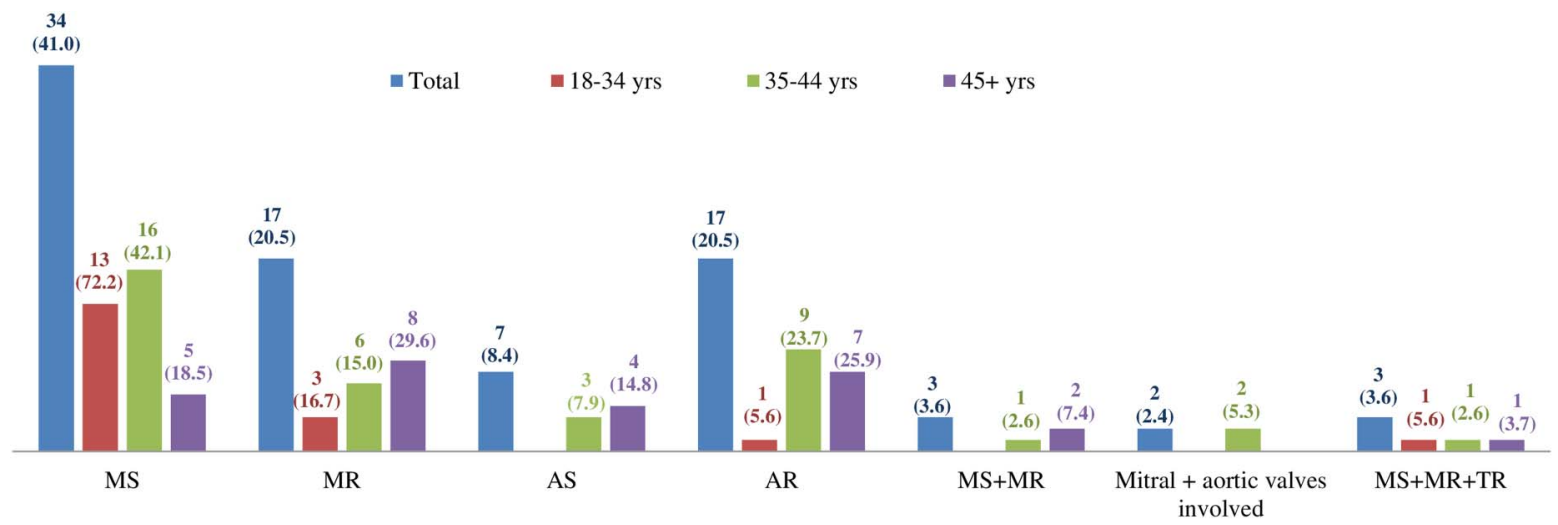

Figure 3 Distribution of valvular heart lesion classifications among cases of rheumatic heart disease $(\mathrm{n}=83$ ). Data shown as raw frequencies with percentages $(n(\%))$, both overall and age stratified. The $p$ value shown is for the $\chi^{2}$ test comparing distributions across age categories. AR, aortic regurgitation; AS, aortic stenosis; MR, mitral regurgitation; MS, mitral stenosis; TR, tricuspid regurgitation.

RHD cases per 10000 population over the study period was 7.79 , with the lowest point observed in 2013 (3.48 per 10000 ). Unfortunately, the current medical record system is limited and does not contain complete data regarding surgical treatment, as all cases are referred and treated off-site.

RHD is one of the most readily preventable chronic diseases. Although echocardiographic screening to detect subclinical disease in endemic communities has been suggested as an effective strategy towards limiting the effects of RHD through early disease altering intervention, ${ }^{21}{ }^{22}$ it is not routinely practised as such at our study sites.

For the $65 \%$ of the workforce diagnosed with RHD who were still employed with the mining company at the conclusion of our review period, medical expenses were covered by the employer. However, for those exiting the workforce, RHD complications could lead to catastrophic healthcare spending. In many cases, employees are sole providers within their household, and severe illness may result in loss of income earning opportunities as well as diversion of productive labour to caring for affected household members, particularly in the latter phases of the disease. In this context, effective population level early prevention and control efforts are especially critical for tackling the burden of RHD.

\section{Study strengths and limitations}

The importance of this study is grounded in the insight it offers into the patterns and prevalence of RHD in a hitherto underexplored geographic region. Moreover, an underlying strength is that diagnosis was based on strict clinical criteria, including echocardiography, meaningful data from which were available for all identified cases. Nevertheless, the findings must be considered in light of several inherent methodological limitations.

First, this was a record based occupational cohort study and thus includes retrospective information pertaining only to individuals employed by PT Freeport Indonesia within the designated 6 year period. Although the data may thus serve as a rough surrogate to describe the magnitude of RHD in the surrounding community, the findings may not be applicable to the general population, particularly in light of the well known 'healthy worker effect', 23 as a result of which the wider population level magnitude and severity of the burden are likely to be underestimated. Secondly, important information, such as surgical treatment, was not included in the records and hence could not be analysed. Moreover, milder cases of valvular disease may have been missed by clinical examination or other diagnostic methods, such as electrocardiography. Similarly, a number of key background and employment history variables were not measured, meaning that meaningful adjusted analyses of vulnerability could not be undertaken. Such underlying determinants must be taken into account, and suitable corrective measures set in place when designing population wide preventive measures.

\section{CONCLUSIONS}

This report provides significant insight into the burden of RHD among a unique occupational cohort in the developing setting of Papua Province, Indonesia. Such studies will continue to be important for describing the burden of an understudied public

\section{Key messages}

What is already known about this subject?

More than 15.6 million people globally suffer from rheumatic heart disease (RHD), and 233000 die prematurely of the disease every year. It is the most important cause of acquired cardiovascular disease in children and young adults in low and middle income countries. However, epidemiological data remain sparse and inconsistent. The political will for funding and implementation of prophylaxis programmes, and the roll-out of cardiac surgery, will depend on better insight into the impact of RHD on population health, productivity and premature mortality.

\section{What does this study add?}

This study provides significant insight into the burden of RHD among an adult cohort in the developing setting of Papua Province, Indonesia. Findings from this occupational cohort highlight that rheumatic fever (RF) and RHD remain major health issues into adulthood. The most common valvular lesion being mitral stenosis, the cumulative RHD risk was significantly higher among older age groups.

\section{How might this impact on clinical practice?} Comprehensive primary and secondary RF/RHD prevention programmes alongside echocardiography screening for school-aged children should be considered in low and middle income country settings. Our study suggests that there may be a role for expanding this screening in occupational settings. Tailored interventions targeting high risk groups, such as older employees, may be especially important in alleviating the RHD burden. 
health challenge. Without a deeper understanding of disease progression and the actual impacts of RHD on affected countries, however, the necessary political pressure for rollout of secondary prophylaxis, medical management and surgical intervention will be insufficient.

RHD will continue to cause high morbidity and mortality in Indonesia and among the world's poorest populations globally unless current prevention initiatives expand and new programmes are established. Concerted efforts should be made to implement comprehensive primary and secondary RF/RHD prevention programmes, and echocardiographic screening for school aged children considered. Our study suggests that there may be a role for expanding this screening in occupational settings. Finally, as the political will for funding and implementation of prophylaxis programmes and cardiac surgery will depend on better insight into the impact of RHD on population health, productivity and premature mortality, further research into the natural history of the disease in the South-East Asia context will be critical.

Twitter Follow Rosemary Wyber at @RosemaryWyber

Acknowledgements We sincerely thank the COPPER study team and all mine employees who made this study possible. We gratefully acknowledge Seno Aji Wibowo and Fadjar Simorangkir for helping to assemble the data.

Contributors RR-F compiled the data, conceived and designed the overall study, and drafted the article. RA analysed the data and contributed to the development and revision of the article. RW, JC and WW contributed to the development and revision of the article. All authors reviewed and approved the final article.

Competing interests None declared.

Provenance and peer review Not commissioned; externally peer reviewed.

Open Access This is an Open Access article distributed in accordance with the Creative Commons Attribution Non Commercial (CC BY-NC 4.0) license, which permits others to distribute, remix, adapt, build upon this work non-commercially, and license their derivative works on different terms, provided the original work is properly cited and the use is non-commercial. See: http://creativecommons.org/ licenses/by-nc/4.01

\section{REFERENCES}

1 Carapetis JR, McDonald M, Wilson NJ. Acute rheumatic fever. Lancet 2005;366:155-68.

2 Carapetis JR. Rheumatic heart disease in developing countries. NEJM 2007:357:439-41.

3 Carapetis JR, Steer A, Mulholland E, et al. The global burden of group A streptococcal disease. Lancet Infect Dis 2005;5:685-94.
4 Zuhlke L, Steer A. Estimates of the global burden of rheumatic heart disease. Global Heart 2013;8:189-95.

5 Murray CJ, Vos T, Lozano R, et al. Disability-adjusted life years (DALYS) for 291 diseases and injuries in 21 regions, 1990-2010: a systematic analysis for the Global Burden of Disease Study 2010. Lancet 2012;380:2197-223.

6 World Health Organization (WHO). The global burden of disease: 2004 update. Geneva: WHO, 2008.

7 Jackson SJ, Steer AC, Campbell H. Systematic review: Estimation of global burden of non-suppurative sequelae of upper respiratory tract infection: rheumatic fever and post-streptococcal glomerulonephritis. Trop Med Int Health 2011;16:2-11.

8 Marijon E, Mirabel M, Celermajer DS, et al. Rheumatic heart disease. Lancet 2012:379:953-64.

9 Shah B, Sharma M, Kumar R, et al. Rheumatic heart disease: progress and challenges in India. Indian J Pediatr 2013;80(Suppl 1):S77-86.

10 Krishna Kumar R, Tandon R. Rheumatic fever and rheumatic heart disease: the last 50 years. Indian J Med Res 2013;137:643-58.

11 Suciadi L, Karwiky G, Hidayat S. PT438 A profile of rheumatic heart disease at a cardiac center in Bandung City, Indonesia: an echocardiography study. Global Heart 2014;9:e258-9.

12 Smith SC, Collins A, Ferrari R, et al. Our time: a call to save preventable death from cardiovascular disease (heart disease and stroke). J Am Coll Cardiol 2012:60:2343-8

13 United Nations Development Programme (UNDP). Papua needs assessment: an overview of findings and implications for the programming of development assistance. UNDP. 2005. http://www.undp.or.id/papua/docs/PNA_en.pdf (accessed 11 Jun 2015)

14 Barold SS, Sischy D, Punzi J, et al. Advanced atrioventricular block in a 39-year-old man with acute rheumatic fever. Pacing Clin Electrophysiol 1998;21(11 Pt 1):2025-8.

15 Arora R, Subramanyam G, Khalilullah M, et al. Clinical profile of rheumatic fever and rheumatic heart disease: a study of 2,500 cases. Indian Heart J 1981:33:264-9.

16 Faheem M, Hafizullah M, Gul A, et al. Pattern of valvular lesions in rheumatic heart disease. J Postgrad Med Inst 2007;21:99-103.

17 Fadahunsi HO, Coker AO, Usoro PD. Rheumatic heart disease in Nigerian children: clinical and preventive aspects. Ann Trop Paediatr 1987;7:54-8.

18 Ravisha MS, Tullu MS, Kamat JR. Rheumatic fever and rheumatic heart disease: clinical profile of 550 cases in India. Arch Med Res 2003;34:382-7.

19 Melka A. Rheumatic heart disease in Gondar college of medical sciences teaching hospital: socio-demographic and clinical profile. Ethiop Med J 1996;34:207-16.

20 Joseph N, Madi D, Kumar GS, et al. Clinical spectrum of rheumatic fever and rheumatic heart disease: a 10 year experience in an urban area of South India. N Am J Med Sci 2013;5:647-52.

21 Manji RA, Witt J, Tappia PS, et al. Cost-effectiveness analysis of rheumatic heart disease prevention strategies. Expert Rev Pharmacoecon Outcomes Res 2013:13:715-24.

22 Zühlke L, Mayosi BM. Echocardiographic screening for subclinical rheumatic heart disease remains a research tool pending studies of impact on prognosis. Curr Cardiol Rep 2013;15:343.

23 McMichael AJ. Standardized mortality ratios and the "healthy worker effect": scratching beneath the surface. J Occup Med 1976;18:165-8. 


\section{Correction}

Rodriguez-Fernandez R, Amiya R, Wyber R, et al. Rheumatic heart disease among adults in a mining community of Papua, Indonesia: findings from an occupational cohort. Heart Asia $2015 ; 7: 1-5$.

The author's name Wishnu Widdodow should read Wishnu Widodo.

Heart Asia 2015;7:19. doi:10.1136/heartasia-2015-010641.corr1

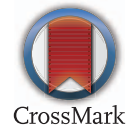

